the other (for example septicaemia due to Neisseria meningitidis or infection with HIV), with examples of infections of every conceivable site, rate, and severity in between.

Upon first consideration, it seems surprising that "we" and "they", driven by the same fundamental aims to survive and multiply, should have thrown up such a myriad of diverse relationships. However, the existence of so many possible distinct mechanisms of transmission and sites of colonisation explains the large number of distinct niches that exist for micro-organisms.

More puzzling is why so many infections are virulent (that is cause disease) at all. Sometimes it is fairly obvious how induction of certain symptoms of disease contributes to the survival and spread of invading micro-organisms (for example diarrhoea in cholera and sneezing in rhinovirus infection). However, a "purpose" for disease features is often less clear. For example it is hard to see how the survival of Streptococcus pneumoniae is enhanced by causing countless episodes of acute ear pain in preschool children and occasional episodes of meningitis. Some well known infectious diseases (for example tetanus and human infections with Pseudomonas spp) would appear to be the results of extraordinary and largely irrelevant excursions into the human for microbes whose modus vivendi is inhabiting the soil and stagnant water.

Of course, as doctors, we tend to focus our attention on disease-producing micro-organisms rather than the many others which have taken an evolutionary stance not unlike that of sheep-achieving enormous success in terms of sheer numbers by being useful, keeping quiet, not causing problems, and putting up with rough treatment at times. Most of us have learned to adopt what could be termed the "microbe centred" view of infectious diseases, namely that these are examples of "invaders" penetrating and colonising the human host and doing damage along the way. As Mitchell's article points out, the manifestations of disease are as often as not due to the host response, or over-response, to the situation.

\section{Growing interest in overgrowth}

Congenital malformations or complex malformation syndromes are frequently associated with growth failure and have been the subject of much research and discussion in the paediatric literature. The less common overgrowth syndromes (OGSs) have until recently received little attention. The disordered growth in OGSs is, however, a primary anomaly and, unlike growth failure, is not explained away as a secondary phenomenon as is the case with many other complex syndromes. OGSs may therefore provide a fascinating window into the mechanisms of growth and the consequences of the failure of this regulation.

Ancient literature has many references to giants such as Goliath, Polyphemus, Gargantua, or the Patagonian giants. Whether real or fictional, these reports show that such patterns have been present throughout history and serve to highlight two of the central issues-what is a "true overgrowth syndrome and how many overgrowth syndromes exist?"

Previously, overgrowth patterns were often categorised as primary or secondary. In primary disorders, the growth would be an intrinsic (unexplained) feature of the condition secondary to cellular hyperplasia, whereas in secondary disorders an identifiable cause, often endocrino-
Clearly eukaryotic organisms evolved a fundamental principle of survival - manifest as the immune responsevery early on, namely: “if it's inside me but isn't me, kill it before it kills me". This process remains the central dogma of our immunity because, despite important exceptions such as mitochondria and long term colonisation with herpes viruses and mycobacterium tuberculosis which (usually) don't do any harm, owners of immune systems which give invading microbes the benefit of the doubt don't survive well.

Our immune responses set clear boundaries to the hoards of microbes that surround us (outside and inside). Our secreted protein and phagocyte defences endeavour to inflict instant death on the invader and our specific immunity acts as a slower but highly organised search and destroy machinery for viruses or larger microbes which succeed in penetrating our initial defences.

Thus, infectious diseases, when they occur, are the result of the interaction between microbe and host. In other words, infection and immunity are two sides of the same coin and cannot be studied legitimately in isolation from each other.

Experience with vaccines has already shown the potential power of inducing host responses in the prevention of infectious diseases. As knowledge of the molecular details of our interactions with micro-organisms continues to grow, this will certainly also lead to new interventions designed specifically to treat disease by modifying the host response or by interfering with microbe-host interactions, as well as to additional conventional agents designed to inhibit or kill the microbe.

ADAM FINN Sheffield Institute for Vaccine Studies, Department of Paediatrics, Sheffield Children's Hospital, Western Bank, Sheffield S10 $2 \mathrm{TH}$

logical, would be expected to result in growth excess. ${ }^{1}$ The limitations of this rather simplistic differentiation have been illustrated by the identification of "novel" growth factors in a number of OGSs, such as Beckwith-Wiedemann syndrome (BWS) and Simpson-Golabi-Behmel syndrome (SGBS). In these disorders, abnormalities of insulin-like growth factor II (IGF II) and glypican 3 have been implicated. ${ }^{2}$ It would seem that if the term secondary growth excess is still relevant, it should be limited to situations dependent on extrinsic growth promoters, such as fetal macrosomia secondary to maternal diabetes and hyperglycaemia and subsequent fetal hyperinsulinaemia. For the foreseeable future most "primary" OGSs will be classified by a process of clinical assessment and/or laboratory exclusion with the possible exceptions of BWS and SGBS, where clinical application of molecular tests might be feasible within a few years.

The constraints of this review preclude the discussion of another group of overgrowth patterns: those syndromes exhibiting regional/tissue specific overgrowth (table 1). These may provide further clues to specific growth promoters, and will have to be considered when developing a model of overall growth control. 


\section{Endocrinological and metabolic disorders with an overgrowth component}

Abnormalities of the hypothalamic pituitary adrenal axis may result in early overgrowth. A number of these are autosomal recessive and the most common is congenital adrenal hyperplasia due to 21-hydroxylase deficiency. In untreated cases, the production of excess 7-hydroxyprogesterone and androgenic steroids results in increased growth from birth and precocious puberty secondary to the anabolic effects of the steroids. ${ }^{4}$ Secretory tumours within this axis may also result in similar overgrowth patterns. A much rarer recessive adrenal disorder is adrenocorticotrophic hormone receptor deficiency. This may present with symptoms suggestive of adrenal failure or overgrowth, or both, and prompt treatment of the adrenal insufficiency is of obvious importance. ${ }^{5}$

The aetiology of the overgrowth and endocrinological confirmation of each of the above is clear. This has not always been the case for disorders with proved endocrinological abnormalities, for example, Seip-Berardinelli lipodystrophy syndrome. This autosomal recessive condition appears to show insulin resistance, but normal pituitary and adrenal function on formal testing. The growth excess can be striking (considerably more than 97th centile), and appears to be more than would be expected from the postulated anabolic component of the syndrome. After some 30 years of research, the mechanism of overgrowth and its link to insulin resistance is only now becoming clearer. ${ }^{6}$

One further condition that may rarely present with overgrowth, which is important not to miss because of the neurological and genetic implications, is the recessive condition, mucopolysccharidosis type III (Sanfilippo's syndrome). ${ }^{7}$ Early literature indicates that the excess growth is only apparent in the first two to three years of life, and that the regression and mental handicap is obvious by this stage. This is in fact frequently incorrect, and I have personal experience of a 6 year old patient over the 90th centile for all growth measurements, who was then only starting to show a decline of intellectual abilities into the range of mild mental handicap.

\section{Congenital malformation syndromes with} overgrowth as the major intrinsic component

The majority of conditions with "primary" overgrowth are poorly understood but are part of an ever increasing list of possible diagnoses. The current London dysmorphology database lists 283 conditions with either macrocephaly, obesity, increased birth weight, or excess stature. ${ }^{8}$ Personal experience in excess of 300 children, is that as many as $50 \%$ do not easily sit within the diagnostic categories currently recognised.

Any diagnostic assessment must include taking an adequate family history and measurements of the nuclear family, as the largest single factor is without doubt familial large stature or early maturation. The presence of some additional sign or symptom within an individual does not necessarily require a syndrome diagnosis because single malformations, or some degree of learning difficulty, is present in up to $10 \%$ of the population. ${ }^{9}$

All possible diagnostic considerations will not be covered within this annotation, however the reader is referred to the excellent review of Cohen. ${ }^{1}$ Tables 1 and 2 (modified from Cole and Hughes ${ }^{10}$ ) also list some of the range of regional and generalised overgrowth disorders.

\section{BWS and SGBS-two OGSs but a common aetiological pathway}

BWS, first described in $1963,{ }^{11}$ has provided much of the recent impetus for research into overgrowth patterns. It has a reported frequency of one in 13700 from a study in
Table 1 Examples of regional or tissue specific overgrowth

Autosomal dominant macrocephaly

Macrocephaly cutis marmorata telangiectasia

Neurofibromatosis

Proteus syndrome

Encephalocraniocutaneous lipomatosis

Hemihypertrophy

Klippel-Trénaunay-Weber syndrome

Maffucci's syndrome

Olliers syndrome

Prader-Willi syndrome

Cohen's syndrome

Carbohydrate glycoprotein deficiency

Fragile X syndrome

Trisomy 8 mosaicism

Jamaica. ${ }^{12}$ I would, however, be concerned of the influence of the high frequency of umbilical hernias (also known to be associated with an increased familial frequency of exomphalos) in this population. ${ }^{13}$ This might have led to over diagnosis, or might indicate that the population as a whole had a higher than average frequency of some of the predisposing mechanisms of BWS.

In BWS, the three main components are abdominal wall defects (exomphalos=E), macroglossia $(\mathrm{M})$, and increased birth weight or growth $(\mathrm{G})$ present in $80 \%, 88 \%$, and $97 \%$ respectively, resulting in the alternative name EMG. ${ }^{14}$ The paediatric relevance of BWS is maintained because approximately $4-7.5 \%$ of patients develop childhood tumours, most commonly Wilms' tumours. ${ }^{114}$ Hemihypertrophy, present in $13-24 \%$ of patients with BWS, ${ }^{14}$ is reported in $40 \%$ of cases with tumours, ${ }^{1}$ therefore the presence of hemihypertrophy appears to be associated with an increased risk of tumourigenesis.

Growth in BWS is usually most marked during the first few years of life. This is compatible with current evidence that IGF II, the likely growth factor in BWS, has its greatest influence during this period. By contrast, final height in many individuals with BWS is within the normal range, although there are exceptions. There is even a suggestion of a bimodal pattern, with a small group showing excessive height. It will be of interest to see if specific molecular mechanisms are associated with different growth patterns.

Despite neonatal symptoms, which can include feeding and respiratory difficulties and very rarely neonatal death, the large majority of survivors have few severe nonmalignant health problems. Intellectual development is believed to show the same distribution as the general population, with the possible exception of patients with extreme or untreated hypoglycaemia or with unbalanced chromosomal translocations.

Of particular genetic interest are the mechanisms underlying BWS. It is estimated that approximately $15 \%$ of families show autosomal dominant inheritance, ${ }^{14}$ and these show linkage to the chromosome region $11 \mathrm{pl} 5.5$, including the insulin IGF II gene loci. ${ }^{15}$ Further studies, reviewed by

Table 2 Generalised overgrowth conditions

Familial/sporadic constitutional gigantism

Familial/sporadic precocious maturation

Bannayan-Riley-Ruvalcaba syndrome

Marfan's syndrome

Beckwith-Wiedemann syndrome

Marshall-Smith syndrome

$\mathrm{MOMO}^{\star}$ syndrome

Nevo's syndrome

Perlman's syndrome

Seip-Barardinelli syndrome

SGBS

SS

WSS

Congenital adrenal hyperplasia

Pituatry/adrenal and gonadal secreting tumours

Sanfilippo's syndrome

Klinefelter's syndrome

${ }^{\star} \mathrm{MOMO}=$ macrosomia-obesity-macrocephaly-ocular anomalies 
Table 3 Comparison of the phenotypes of BWS and SGBS

\begin{tabular}{lll}
\hline & $B W S$ & $S G B S$ (in boys) \\
\hline Macroglossia & ++ & ++ \\
Abdominal wall defects & ++ & + \\
Visceromegaly & ++ & ++ \\
Hypoglycaemia & ++ & + \\
Embryonal tumours & $+/(+)$ & $+/(+)$ \\
Ear creases & ++ & ++ \\
Ear pits & + & $?$ \\
Flammeus naevus & ++ & ++ \\
Cleft lip/palate & $(+)$ & + \\
Groved tongue/lip & $?$ & ++ \\
Facial outline & Round & Square \\
Coarsening facial gestalt & + & ++ \\
Genital anomalies & $(+)$ & + \\
Congenital heart disease & $(+)$ & + \\
Additional nipples & $?$ & + \\
Postaxial polydactyly & $(+)$ & + \\
Syndactyly & $(+)$ & + \\
Short/squared distal phalynx & $?$ & ++ \\
Final height increased & + & ++ \\
\hline Approximate frequency of anomalies: & $++>50 \%,+5-50 \%,(+)<5 \%, ?$ frequency \\
unclear but probably rare or absent. & &
\end{tabular}

Mannens et al, showed that paternal duplications, maternal translocations, and paternal uniparental disomy (the presence of only paternal chromosome material) involving $11 \mathrm{pl} 5.5$ result in BWS. ${ }^{16}$ There is evidence that the phenotype results from overexpression of IGF II, a gene usually only expressed on chromosomes of paternal origin. ${ }^{2}$ Recent papers have shown that mutations within $\mathrm{p} 57^{\mathrm{KIP} 2},{ }^{17}$ or disruption of the gene KVLQT $1,{ }^{18}$ which both map proximal to IGF II at $11 \mathrm{pl} 5.5$, will result in the BWS phenotype. In both instances the maternally inherited gene is affected. At least one family with a translocation disrupting KVQLT1 had biallic IGF II expression, while p $57^{\mathrm{KIP} 2}$ may be involved in a common pathway with IGF II. A p $57^{\mathrm{KIP} 2}$ gene "knockout" mouse, ${ }^{19}$ and subsequent mutation studies of KIP2 in humans, also results in a BWS phenotype, but once again only if the maternal allele is affected. ${ }^{20}$ There also appears to be some early evidence for genotype/phenotype correlations with $\mathrm{p} 57^{\mathrm{KIP} 2}$ mutation carriers having a high frequency of abdominal wall defects (five out of seven had exomphalos, one out of seven had an umbilical hernia) but no embryonal tumours, ${ }^{20}$ whereas uniparental disomy $11 \mathrm{pl} 5.5$ may be associated with a slightly greater risk of tumourigenesis. ${ }^{16}$

This phenomenon of imprinting (the regulation of gene expression dependent on parent of origin) is believed to be important in numerous different conditions with disordered growth including Prader-Willi syndrome, Angelman's syndrome, Russell-Silver syndrome, and certain tumours, and has therefore been the subject of intense investigation. $^{2122}$

During research into BWS, clinicians noted the striking phenotypic overlap with SGBS, an X linked overgrowth disorder (table 3). ${ }^{23}{ }^{24}$ In the report of Thorburn et al the diagnosis of SGBS in some, or all, of the three boys (all of whom died in infancy and had additional malformations recognised in SGBS) remains a possibility, and is one further source of error which could result in overestimation of the incidence in BWS in their paper. ${ }^{12}$

After the identification of glypican 3 mutations as the cause of SGBS, ${ }^{3}$ subsequent antibody studies showed there was cross reaction of binding between the ligands for glypican and IGF II receptors. ${ }^{325}$ This in turn might explain the striking phenotypic overlap and also raised the possibility of further diagnostic errors in the literature or clinical setting. The latter has significant implications for genetic counselling.

An additional consideration is the existence of an undiagnosed group of patients, as reported in the paper by Morrison et al. $^{26}$ They described "non"-BWS patients with overexpression of IGF II. I would suggest that the presence of overgrowth and nephromegaly in these patients might represent the mild end of the spectrum of BWS. An alternative explanation is that many macrosomic babies have some underlying mechanism which results in abnormalities of IGF II expression, the BWS phenotype being just one of the resultant outcomes. If the outcome is not the BWS phenotype, it raises the fascinating possibility that overexpression of IGF II might explain the excess of neuroblastoma and Wilms' tumours in babies weighing over $4000 \mathrm{~g} .{ }^{27}$ As we start to unravel the mechanisms behind BWS and SGBS it appears that a more intriguing set of questions are raised.

While molecular studies for glypican 3 and IGF II are still at an early stage, it seems likely that within the next 2-3 years these will become part of the routine investigation for BWS and SGBS in an effort to confirm the diagnosis and the recurrence risk, and perhaps even help predict the individual natural histories.

Sotos' syndrome (SS), Weaver-Smith syndrome (WSS), and other overlapping phenotypes

My own experience, and that within our department, is that referrals of SS and BWS are approximately equal although this figure may be biased by my own research interests. However, it is probable that SS is at least the second most common overgrowth disorder after BWS.

SS was first reported in $1964,{ }^{28}$ although I suspect the first literature case may be the report of Schlesinger in 1932. ${ }^{29}$ The clinical features are of prenatal and postnatal overgrowth, developmental delay, characteristic facial appearance, and an advanced bone age. Apart from the subjective assessment of facial gestalt (table 4) and relative frequencies of developmental delay, the remaining diagnostic criteria are not only features of BWS and SGBS, but also the WSS and Marshall-Smith syndrome (MSS). ${ }^{30} 31$ Furthermore, a number of less frequent signs such as hernias, minor skeletal anomalies, and tumour predisposition are probably present to varying degrees in each of the above disorders. Currently the majority of opinion splits SS, WSS, and MSS into three distinct syndromes.

The growth in SS is most marked during the first 4-5 years of childhood, although may be "masked" during the first 36 months of life if complications secondary to prematurity, hypotonia, poor feeding, or respiratory difficulties are present. The two growth variables most significantly increased are length and occipitofrontal circumference, whereas weight is often lower than would be expected for the patients stature. ${ }^{32}$ Final height is commonly below or close to the $+2 \mathrm{SD}$. This appears to be

Table 4 Facial gestalt of SS, WSS, and MSS

SS

Dolichocephaly

Tall bossed forehead

High frontal hairline

Slow/sparse hair growth fronto-parietal areas

Pear shaped facial outline

Lengthening face in childhood

Long jaw

Down slant palpebral fissures

Red facial colouring

WSS

Broad rounded forehead

Round facial outline

Small prominent chin

Rounded full mid face

Long prominent philtrum

Large ears

MSS

Fine facial bones

Prominent eyes

Thin gaunt facies

Anteverted nares

Micrognathia 
particularly true of girls whose growth often stops earlier than their peers after a relatively early puberty. This pattern appears more variable in boys, but still very few exceed or even approach $+3 \mathrm{SD}$ (Agwu et al in preparation). It is the authors experience that while careful monitoring of growth, bone age, and pubertal status is advisable, therapeutic intervention is almost never necessary to obtain an "acceptable" final height.

SS was originally incorrectly reported to have a high frequency of mental handicap (88\%). ${ }^{33}$ It is now clear that the figure is lower, and Finegan et al reported that $78 \%$ of children have an IQ above $70 .^{31}$ This still remains one of the highest figures for OGSs and is often associated with significant behavioural problems. ${ }^{34}$ Other significant medical complications are individually uncommon. ${ }^{32}$

Despite the similarities with WSS and MSS, these two conditions appear to exhibit higher frequencies of severe medical complications, including skeletal anomalies and infant death. Surprisingly, personal experience of the three older cases of WSS (two adults and one adolescent) and the two oldest literature cases of MSS is that intellectual abilities have been within, or near to, the normal range in all these individuals. ${ }^{35}$ This might be fortuitous, as significant intellectual impairment has been documented in both disorders.

The range of final heights in WSS remains unclear, however three literature reports of adults with WSS, and my own experience of two adult patients, may suggest that heights, significantly in excess of $+2 \mathrm{SD}$, are more common than in SS. ${ }^{30}{ }^{32}$ It should be stressed that ascertainment bias could be very relevant in four out of five of these cases.

\section{The genetics of OGSs}

The genetics of BWS is that the majority are sporadic, but approximately $15 \%$ follow autosomal dominant inheritance. In my experience of approximately 200 cases of SS, three cases, and one of 15 cases of WSS segregated in an autosomal dominant fashion, ${ }^{30}$ the remainder are sporadic. Unlike BWS, to date there has been no consistent region of chromosomal alteration in the other OGSs, and a study of uniparental disomy in SS was negative within the limitations of the study. ${ }^{37}$ The genetics therefore remain obscure in the majority of OGSs.

The clinical similarities among the OGSs should intrigue, yet alert, the researcher to the issue of whether the OGSs should be "lumped" or "split". It seems certain that only as the molecular basis unfolds will it become clear if the disorders are differing ends of the same spectrum or separate conditions. Could it be that they will be allelic variations in the same gene, such as in the RET oncogene which results in the different multiple endocrine neoplasia II syndromes and Hirschsprung's disease, ${ }^{38}$ or mutations in the same fibroblast growth factor gene associated with the different craniosynostosis syndromes such as Apert's, Pfeiffer's, Crouzon's, and Jackson-Weiss? ${ }^{39}$ Or perhaps the different OGSs will be due to mutations within a group of genes like the fibroblast growth factor I, II, and III genes, each associated with syndromes including the feature of craniosynostosis, ${ }^{39}$ or the different fibrillin genes which are the cause of Marfan's syndrome (fib 1), ${ }^{40}$ congenital contractural arachnodactyly (fib 2), ${ }^{41}$ and ShprintzenGoldberg (fib 1). ${ }^{42}$ While these are possible mechanisms, the only precedent in OGSs is in BWS and SGBS. In these two conditions, none of the above mechanisms apply, but rather two different genes interact on the same pathway. ${ }^{25}$
Different overgrowth patterns-different genetic mechanisms

AUTOSOMAL DOMINANT MACROCEPHALIES

The autosomal dominant macrocephalies are one further overgrowth spectrum which appear to show a perplexing degree of pleiotropy and heterogeneity. ${ }^{43}$ They may also overlap with intriguing sporadic macrocephaly conditions such as the macrocephaly, telangiectasia, and cutis mamorata syndrome. Unravelling the mechanisms behind features associated with macrocephaly, such as vascular anomalies ${ }^{43}$ or autism, ${ }^{44}$ could be a further fruitful area of research related to overgrowth disorders.

MOSAIC DISORDERS

This text so far has only considered conditions that I believe to be non-mosaic mutations. Disorders such as Proteus' syndrome and McCune-Albright syndrome, are most likely, or have already been proved to be, due to somatic mutations, and if present in a non-mosaic syndrome it is postulated that they would be lethal in most situations.

Molecular investigations of patients with McCuneAlbright syndrome have confirmed the presence of mosaic mutations in Gs $\alpha$ gene. ${ }^{45}$ Patients with McCune-Albright syndrome show quite remarkable early overgrowth. Much of this appears to be secondary to the effects of a strikingly early puberty. It is clear, however, that the $G$ proteins are a family of signal transducers and have a regulatory role on many different endocrinological and growth factor pathways. The growth and endocrine role and the recognition of de novo mutations makes this group of genes interesting potential candidates for OGSs such as SS and WSS.

\section{Conclusions}

OGSs offer the opportunity to study prenatal and postnatal growth in normal tissues, and may show how abnormal growth may predispose to tumour development. It is also of great interest that many of the genes involved in growth disorders are regulated and (for example, imprinted) dependent upon their parent of origin, which may be highly significant in fetal growth.

The recognition of new OGSs is still occurring and yet as many as $50 \%$ of cases do not appear to fall neatly into any recognised patterns. However, the aetiology of the clinical overlap between diagnosed and currently undiagnosed syndromes may become apparent as the underlying genetic mechanisms become clearer. As this molecular understanding remains incomplete, it is important to try and identify on clinical grounds the specific "syndrome pattern" where possible, as the natural history and genetics may vary considerably.

The author would like to acknowledge the support provided by Action Research which enabled collection of much of his data on overgrowth syndromes and stimulated a lasting interest and Professor Eamonn Maher for his many helpful comments on the annotation.

West Midlands Regional Clinical Genetics Service,

TREVOR COLE

Clinical Genetics Unit,

Birmingham Women's Hospital NHS Trust,

Edgbaston,

Birmingham B15 2TG

1 Cohen MM Jr. A comprehensive and critical assessment of overgrowth and overgrowth syndromes. In: Harris H, Hirschhorn K, eds. Advances in human genetics. New York: Plenum Press, 1989;18:181-303.

2 Weksberg R, Shen DR, Fei YL, Song OL, Squire J. Disruption of insulin like growth factor II imprinting in Beckwith-Wiedemann syndrome. Nat Genet 1993;5:143-50.

3 Pilia G, Hughes-Benzie RM, Mackenzie AK, et al. Mutations in GPC3, a glypican gene, cause the Simpson-Golabi-Behmel overgrowth syndrome. Nat Genet 1996;12:241-7.

4 Donohue PA, Parker K, Migeon CJ. Congenital adrenal hyperplasia. In: Scriver CR, Beaudet AL, Sly WS, Valle D, eds. 7th Ed. The metabolic and molecular basis of inherited disease. New York: McGraw-Hill, 1995;2:2929-66. 
5 Weber A, Toppari J, Harvey RD, et al. Adrenocorticotrophin receptor gene mutations in familial glucocorticoid deficiency: relationship with clinical features in four families. F Clin Endocrinol Metab 1995;80:56-71.

6 Seip M, Trygstad O. Generalised lipodystrophy, congenital and acquired (lipoatrophy). Acta Paediatr Suppl 1996;413:2-28.

7 Neufeld EF, Muenzer J. The mucopolysaccharidoses. In: Scriver CR, Beaudet AL, Sly WS, Valle D, eds. 7th Ed. The metabolic and molecular basis of inherited disease. New York: McGraw-Hill, 1995;2:2465-94.

8 Winter RM, Baraitser M. London dysmorphology database. Oxford: Oxford University Press, 1993

9 Rimoin DL, Connor JM, Pyeritz RE. Nature and frequency of genetic disease. In: Rimoin DL, Connor JM, Pyeritz RE, eds. 3rd Ed. Emery and Rimoin's principles and practice of medical genetics. New York: Churchill Livingstone, 1996;1:31-4.

10 Cole TRP, Hughes HE. Sotos syndrome. F Med Genet 1990;27:571-6.

11 Beckwith JB. Extreme cytomegaly of the adrenal fetal cortex, omphalocele, hyperplasia of the kidneys and panceas, and Leydig-cell hyperplasia: another plasia of the kidneys and panceas, and Leydig-cell hyperplasia: another
syndrome? Presented at the Annual Meeting of the Western Society for syndrome? Presented at the Annual
Pediatric Research, Los Angeles, 1963.

12 Thorburn MJ, Wright ES, Miller CG, Smith-Read EHM. Exomphalosmacroglossia-gigantism syndrome in Jamaican infants. Am $\mathcal{f}$ Dis Child 1970;119:316-21.

13 Evans AG. Comparative incidence of umbilical hernias in colored and white infants. F Natl Med Assoc 1944;33:158

14 Elliott M, Bayly R, Cole T, Temple IK, Maher ER. Clinical features and natural history of Beckwith-Wiedemann syndrome: presentation of 74 new cases. Clin Genet 1994:46:168-74.

15 Koufos A, Grundy P, Morgan K, et al. Familial Wiedemann-Beckwith syndrome and a second Wilms' tumour locus both map to $11 \mathrm{pl5}$.5. Am f Med Genet 1989;44:711-9.

16 Mannens M, Hoovers J, Redeker E, et al. Parental imprinting of human chromosome region 11pl5.3-pter involved in the Beckwith-Wiedemann syndrome and various human neoplasia. Eur 7 Hum Genet 1994;2:3-23.

17 Hatada I, Ohashi H Fukushima Y. An imprinted gene p $57^{\mathrm{KIP} 2}$ is mutated in Beckwith-Wiedemann Syndrome. Nat Genet 1996;14:171-3.

18 Lee MP, Hu R-J, Johnson LA, Feinberg AP. Human KVLQT1gene shows tissue-specific imprinting and encompasses Beckwith-Wiedemann syntissue-specific imprinting and encompasses Beckwith-Wiedem
drome chromosomal rearrangements. Nat Genet 1997;15:181-5.

19 Pumin Z, Liegeois NJ, Wong K, et al. Altered cell differentiation and proliferation in mice lacking p57kip2 indicates a role in Beckwith-Wiedemann eration in mice lacking p57kip2 indicise.

20 Lam WWK, Hatada I, Ohishi S, et al. Analysis of germline p57KIP2 mutations in the imprinting disorder Beckwith-Wiedemann syndrome (BWS) provides a genotope-phenotype correlation. Am F Hum Genet 1997;61: (suppl 3)

21 Clarke A. Genetic imprinting in clinical genetics. Development 1990;(suppl): 131-9.

22 Feinberg AP. Geneomic imprinting and gene activation in cancer. Nat Genet 1993;4:110-3

23 Hughes-Benzie RM, Hunter A, Allanson J, Mackenzie A. Simpson-GolabiBehmel syndrome associated with renal dysplasia and embryonal tumour: localisation of a gene to Xqcen-q21. Am f Med Genet 1992;43:428-35.

24 Hughes-Benzie RM, Allanson J, Hunter A, Cole T. The importance of differentiating Simpson-Golabi-Behmel and Beckwith-Wiedemann syndromes. F Med Genet 1992;29:228.
25 Weksberg R, Squire JA, Templeton DM. Glypicans: a growing trend. Nat Genet 1996;12:225-27.

26 Morison IM, Becroft DM, Taniguchi T, Woods CG, Reeve AE. Somatic overgrowth associated with overexpression of insulin-like growth factor II. Nat Med 1996;2:311-16.

27 Daling JR, Starzyk P, Olshan AF, et al. Birth weight and the incidence of childhood cancer. F Natl Cancer Inst 1984;72:1039-41.

28 Sotos JF, Dodge PR, Muirhead D, Crawford JD, Talbot NB. Cerebral gigantism in childhood. $N$ Engl f Med 1964;271:109-16.

29 Schlesinger B. Gigantism (acromegalic in nature). Proc R Soc Med 1931;24: 1352

30 Cole TRP, Dennis NR, Hughes HE. Weaver syndrome: seven new cases and a review of the literature. In: Donnai D,Winter RM eds. Congenital malformation syndromes. London: Chapman Hall, 1994:267-80.

31 Fitch N. The syndromes of Marshall and Weaver. 7 Med Genet 1980;17:174-8

32 Cole TRP, Hughes HE. Sotos syndrome: a study of the diagnostic criteria and natural history. $\mathcal{F}$ Med Genet 1994;31:20-32.

33 Jaeken J, Schueren-Lodeweyckx MVAN, Eeckels R. Cerebral gigantism syndrome: a report of four cases and review of the literature. Zeitschrift fur Kinderheilkunde 1972;112:332-46.

34 Finegan JK, Cole TRP, Kingwell E, Smith ML, Smith M, Sitarenioe G. Language and behaviour in children with Sotos syndrome. $\mathcal{F}$ Am Acad Child Adolesc Psychiatry 1994;33:1307-15.

35 Hoyme HE, Byers PH, Guttmacher AK. Marshall-Smith syndrome: further evidence of an osteochondroplasia in long-term survivors. Proceedings of the Greenwood Genetics Center 1993;12:70.

36 Fryer A, Smith C, Rosenbloom L, Cole TRP. Autosomal dominant inheritance of Weaver syndrome. $\mathcal{F}$ Med Genet 1997;34:418-19.

37 Smith M, Fullwood P, Qi Y, Palmer S, Upadhyaya M, Cole T. No evidence for uniparental disomy as a common cause of Sotos' syndrome. 7 Med Genet 1997;34:10-12.

38 Van Heyningen V. One gene-four syndromes. Nature 1994;367:319-20.

39 Mulvihill JJ. Craniofacial syndromes: no such thing as a single gene disease. Nat Genet 1995;9:101-3.

40 Dietz HC, Cutting GR, Pyeritz RE, et al. Marfan syndrome caused by a recurrent de novo missense mutation in the fibrillin gene. Nature 1991;352:337-9.

41 Putnam EA, Zhang H, Ramirez F, Milewicz D. Fibrillin-2 (FBN2) mutations result in the Marfan-like disorder, congenital contractural arachnodactyly. Nat Genet 1995;11:456-8.

42 Sood S, Eldahah ZA, Krause WL, McIntosh I, Dietz HC. Mutations in Fibrillin 1 and the marfanoid-craniosynostosis (Shprintzen-Goldberg) syndrome. Nat Genet 1996;12:209-11.

43 Stephan MJ, Hall BD, Smith DW, Cohen MM. Macrocephaly in association with unusual cutaneous angiomatosis. Pediatrics 1975;87:353-9.

44 Stevenson RE, Schroer RJ,Skinner C, Fender D, Simensen RJ. Autism and macrocephaly. Lancet 1997;349:1744-5.

45 Schwindinger WF, Francomano CA, Levine MA. Identification of a mutation in the gene encoding $\alpha$ subunit of the stimulatory $\mathrm{G}$ protein of adenyl cyclase in McCune-Albright syndrome. Proc Natl Acad Sci USA 1992;89:5152-6.

\section{Diagnosing Friedreich's ataxia}

The condition that now bears his name was first described by Nicolaus Friedreich in a series of papers between 1863 and 1877 . He noted the onset at around puberty of ataxia and dysarthria; sensory loss and weakness developed later. The skeletal deformities of pes cavus and scoliosis were also reported. Over the following generations there was a tendency to lump the inherited ataxias together, and the essential features of Friedreich's ataxia became diluted. Clinical studies in the 1970 s and $1980 \mathrm{~s}^{12}$ and subsequent genetic studies have helped clarify these features, and Friedreich's ataxia is now known to be the commonest of the inherited ataxias, accounting for at least $50 \%$ in most large series and affecting approximately one in 50000 individuals. ${ }^{3}$ Although at present it is an incurable and progressive disease, recent identification of the affected gene has not only provided a highly sensitive and specific diagnostic test, but has also given useful insight into the cellular pathology which may lead to the development of effective treatment.

\section{Clinical diagnosis}

Despite the relatively homogeneous clinical picture of an early onset of progressive ataxia involving the trunk and the limbs, it was necessary to formulate strict clinical criteria in order to perform genetic linkage analyses, and two notable studies, ${ }^{12}$ provided these. Harding's criteria (table 1) were widely adopted and are still useful today, although of course we are now able to reinterpret "atypical" cases in the light of available genetic data.

In addition to ataxia, there are several variable features, including pyramidal tract involvement. Initially this may be mild, with only extensor plantar responses, but after five or more years a pyramidal type of weakness in the legs invariably occurs and this can eventually lead to paralysis. The association of extensor plantar responses, absence of ankle reflexes, and a progressive course provide the core features.

Skeletal abnormalities are also commonly found. These include scoliosis (85\%), and foot deformities; although pes cavus is the best known of these, pes planus and equinovarus are also often found. Amyotrophy of the lower leg and rarely of the hands may also be found. When all these features are present in a case of early onset (before 20 years of age) autosomal recessive ataxia, genetic analysis will prove that Friedreich's ataxia is the correct diagnosis in the vast majority. ${ }^{4}$ Additional clinical support for a suspected diagnosis includes optic atrophy, which occurs in $25 \%$ of cases; however, it is rare for there to be major visual impairment in Friedreich's ataxia (less than $5 \%$ ). ${ }^{2}$ Deafness is found in less than $10 \%$ of cases. 